\title{
PHARMACODYNAMIC AND METABOLISM STUDIES ON A NEW CORONARY VASODILATOR, N-(2-HYDROXYETHYL) NICOTINAMIDE NITRATE (SG-75)
}

\author{
Kazushige SAKAI, Yasuhiro OHBA, Michitaka AKIMA, \\ Hiroshi KAMIYAMA*, Yoshikazu HINOHARA* and Hideki NAKANO* \\ Department of Pharmacology and *Drug Metabolism, Research Laboratories, \\ Chugai Pharmaceutical Co., Ltd., Toshima-ku, Tokyo 171, Japan
}

Accepted June 24, 1980

\begin{abstract}
Pharmacodynamics and metabolism of N-(2-hydroxyethyl) nicotinamide nitrate (SG-75) were investigated in rats in relation to its main metabolic product. When SG-75 was orally administered, SG-75 and SG-86, a denitrate compound of SG-75, appeared in the blood. Within $7 \mathrm{hr}$, approximately $60 \%$ of the administered SG-75 were recovered in the urine as SG-86. When administered intraduodenally, SG-75 was rapidly absorbed and transferred in an unaltered form into the portal vein. SG-75 possessed hypotensive and coronary vasodilating actions, while SG-86 had little effect on the cardiovascular system. The coronary vasodilating effects of SG-75 $(0.3-30 \mu \mathrm{g}$ i.a.) were unaffected by the continuous infusion of SG-86 into the coronary perfusion system, even in large doses $(50 \mu \mathrm{g} / \mathrm{min}$, or $500 \mu \mathrm{g} / \mathrm{min})$.
\end{abstract}

$\mathrm{N}$-(2-hydroxyethyl)nicotinamide nitrate (SG-75) shown in Fig. 1 is a new coronary vasodilator reported by Uchida et al. (1). This compound eliminates cyclic reduction of coronary blood flow and ST-elevation of ECG caused by partial occlusion of the left anterior descending coronary artery in dogs (2). Recently, it has been further demonstrated that SG-75 significantly increases coronary blood flow, virtually without affecting myocardial oxygen consumption and atrio-ventricular conduction in anesthetized, open-chest dogs (3) and canine heart-lung preparations (4). Thus, SG-75 seems to possess the pharmacological characteristics to be an effective antianginal agent. The metabolic fate, particularly in the intestinal tract and tissue has heretofore not been investigated.

The present paper describes quantitative investigations on the metabolic fate of SG-75 in rats, with special reference to pharmacological actions of its main metabolite, SG-86 (see Fig. 1), on the cardiovascular system. A vascularly perfused, isolated intestinal preparation of the rat (5) was utilized.

\section{MATERIALS AND METHODS}

Male Sprague-Dawley rats were used. Unless otherwise stated, the animals were allowed free access to food and water overnight prior to experiments.

Administration and assay of $S G-75$ and its main metabolite, $S G-86$

1. Whole animals: Twenty-seven rats weighing 330-410 g were deprived of food overnight, but water was allowed ad libitum. The animals were given SG-75 p.o. $(5 \mathrm{mg} /$ $10 \mathrm{ml} \mathrm{kg}-1$ as SG-75 solution) and were separated into groups of nine. Laparotomy was 
carried out with rats under ether anesthesia and the blood samples $(5 \mathrm{ml})$ were withdrawn from the inferior vena cava into heparinized syringes, at different time intervals, prior to dosing and at 5, 15, 30 min and 1, 2, 4, 6 and $8 \mathrm{hr}$ following administration of the drug. Plasma was separated immediately, transferred to test tubes and frozen at $-20^{\circ} \mathrm{C}$ until assay.

Two $\mathrm{ml}$ of the plasma was transferred to $15 \mathrm{ml}$ stoppered conical tip glass tubes to which one $\mathrm{ml}$ distilled water and one $\mathrm{ml}$ internal standard solution (one $\mathrm{ml}$ distilled water contained $2.5 \mu \mathrm{g}$ of SG-89 shown in Fig. 1), two drops of $1 \mathrm{~N}-\mathrm{NaOH}$, and $6.5 \mathrm{ml}$ ethyl acetate had been added. The preparation was shaken vigorously for $10 \mathrm{~min}$. The upper ethyl acetate layer was removed after centrifuging at $3 \times 10^{3} \mathrm{rpm}$ for $10 \mathrm{~min}$ and this extraction procedure was repeated. The ethyl acetate layers were combined, transferred to $30 \mathrm{ml}$ pear-shaped glass flask, and evaporated to dryness. Immediately after evaporation, $0.5 \mathrm{ml}$ of distilled water was added to dissolve the residue.

Ten microliters of samples were injected into a high pressure liquid chromatograph (HPLC) system (Waters Associates, Model-6000A) with the $300 \mathrm{~mm}$ stainless steel column of $4 \mathrm{~mm}$ I.D. packed with $\mu$ Bondapac $\mathrm{C}_{18}$. Other operating conditions were as follows: flow rate of the solvent $(25 \%$ methyl alcohol- $7 \%$ ethyl alcohol in distilled water), $1.1 \mathrm{ml} /$ min; operation temperature, $25 \pm 2{ }^{\circ} \mathrm{C}$-room temperature; detector, $254 \mathrm{~nm}$ ultraviolet absorption detector -0.01 absorbance unit full scale; chart speed, $1 \mathrm{~cm} / \mathrm{min}$. Under these conditions, the retention times are 6.0 and $3.5 \mathrm{~min}$ for SG-75 and SG-86, respectively.

Standard curves of SG-75 and SG-86 were prepared as follows: one $\mathrm{ml}$ of a solution which contained the same amount of both SG-75 and SG-86, 0.67, 1.0, 2.0 and $4.0 \mu \mathrm{g}$, respectively, in distilled water, and one $\mathrm{ml}$ of a solution of internal standard, SG-86 $(2.5 \mu \mathrm{g})$
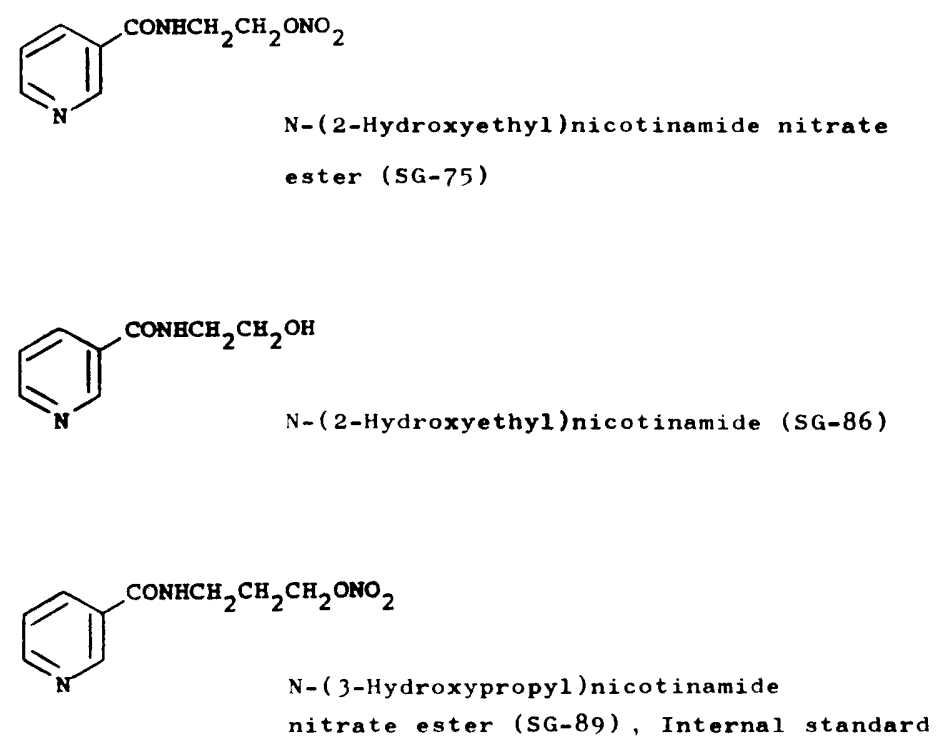

Fig. 1. Chemical structure of SG-75, SG-86 and SG-89. 
<smiles>O=C(N=CC=CCO[N+](=O)[O-])c1cccnc1</smiles>

FIG. 2. Chemical structure and labeled position of ${ }^{14} \mathrm{C}-\mathrm{SG}-75$.

were added to control plasma samples $(2.0 \mathrm{ml})$ to give duplicate standards containing 0.67 , 1.0, 2.0 and $4.0 \mu \mathrm{g}$ of SG-75 and SG-86, respectively. An equal volume of distilled water was added to the control plasma (standard zero). The standard plasma samples so prepared were treated in the same manner as the test samples.

In some experiments, one $\mathrm{mg} / \mathrm{kg}$ of ${ }^{14} \mathrm{C}$-SG-75 (Fig. 2) containing $2.8 \mu \mathrm{Ci}$ of radioactivity dissolved in $0.9 \%$ saline solution was given p.o. to 2 rats fasted for $24 \mathrm{hr}$ prior to the experiment, and urine was collected during $7 \mathrm{hr}$ after dosing. SG-75 and the metabolites in the urine were separated on a $5 \times 20 \mathrm{~cm}$ silica gel layer $(\mathrm{F}-254,0.25 \mathrm{~mm}$ thick, E. Merck) using benzene/EtOH $(4: 1)$. Location of the radioactivities and the UV-absorptive spots on the above thin-layer plate was detected using a Aloka thin-layer chromatograph scanner (Model, TRM-1B), and by UV lamp ( $\lambda=2537 \AA$ ), respectively.

2. Vascularly perfused, isolated intestine of the rat: After fasting overnight, rats weighing $130-150 \mathrm{~g}$ were anesthetized with pentobarbital sodium $(65 \mathrm{mg} / \mathrm{kg}$ i.p. $)$ and the small intestine was isolated, as described elsewhere (5). Briefly, the isolated small intestine was perfused with whole rat blood recycled from a devised oxygenator-reservoir at a fixed flow rate through the superior mesenteric artery. Venous blood from the portal vein was returned to the reservoir portion of a glass oxygenator-reservoir by gravity. Fifteen intestinal preparations were separated into groups of three and in each group $100 \mu \mathrm{g}, 500 \mu \mathrm{g}$ and $1 \mathrm{mg}$ of SG-75, respectively, were given into the duodenum; SG-75 was dissolved in distilled water at appropriate concentrations and with a syringe was given in a volume of $0.2-0.4 \mathrm{ml}$ by direct puncture at the origin of duodenum inside the isolated intestinal tract. The arterial and venous blood $(0.8 \mathrm{ml})$ were concomitantly collected in syringes at $2,5,10$, 20,30 and $60 \mathrm{~min}$ following administration of SG-75, and the plasma $(0.4 \mathrm{ml})$ was immediately separated. The concentrations of SG-75 and SG-86 in these plasma specimens were determined according to the same procedure as described in "whole animals" section.

\section{Cardiovascular studies}

Isolated donor-perfused rat heart: Details were as reported previously elsewhere (6). Briefly, rats were anesthetized with pentobarbital sodium $65 \mathrm{mg} / \mathrm{kg}$ i.p. The isolated heart (left in situ) of the recipient animal (about $300 \mathrm{~g}$ ) was perfused at a fixed flow rate through the coronary arteries via the aorta of the recipient with heparinized blood $\left(37^{\circ} \mathrm{C}\right)$ from the carotid artery of a donor, with the aid of a peristaltic pump (Mitsumi Science, SJ-1210). Output of the pump was precalibrated and re-checked at the end of the experiment. Mean perfusion pressure was recorded near the aortic cannula with a pressure transducer (Nihon Kohden, MPU-0.5). Left ventricular systolic pressure (LVP) was measured isovolumically in the following way. A concentric double-wall cannula, as illustrated in Fig. 3, composed of polyethylene outer and inner tubing was used. A thin latex rubber balloon was attached 

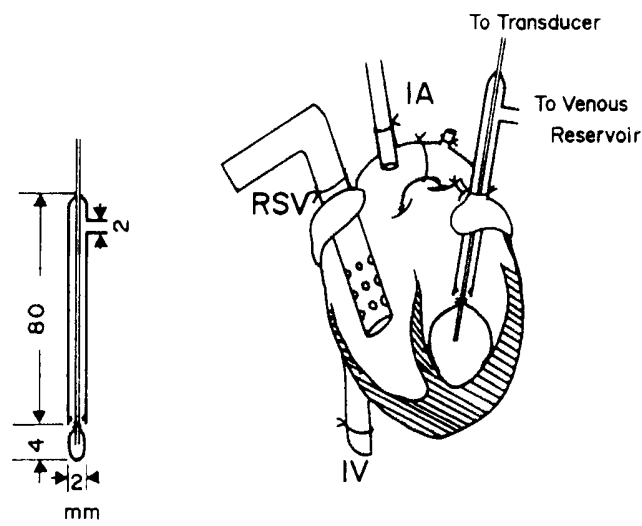

FIG. 3. Arrangement of a concentric double-wall cannula (see Methods section) in the isolated rat heart. RSV, right superior vena cava: IV, inferior vena cava; IA, innominate artery.

to one end of the inner tubing and the other end was connected via a three-way stopcock to a pressure transducer (Nihon Kohden, MPU-0.5) and to a syringe used to fill the balloon and the entire inner tubing with $0.9 \%$ saline. The cannula was introduced into the left ventricular cavity through the left atrium and the mitral valve, and secured tightly using a purse string suture around the cannula at the level of the mitral valve. The balloon was inflated with a sufficient amount of $0.9 \%$ saline (approx. $0.03 \mathrm{ml}$ ) from the syringe to produce LVP of about $100 \mathrm{mmHg}$, and LVP was recorded isovolumically with a pressure transducer (Nihon Kohden, MPU-0.5). The outer tubing had 2-3 pores near the end inserted into the left ventricular cavity and drained a small quantity of blood that leaked through the aortic valve and that drained from the Thebesian veins. This blood was returned to the donor via a venous reservoir. The heart rate was measured by means of a heart rate counter (Nihon Kohden, AT-600G). Recordings were made on an ink-writing recorder (Nihon Kohden, WI-680G).

Drugs were dissolved in $0.9 \%$ saline and the solution in a volume of $0.01 \mathrm{ml}$ was injected into the perfusion system immediately distal to the aortic cannula over a period of $4 \mathrm{sec}$ by means of individual microsyringes (Jintan Terumo Co.). Infusion of the drug solution was given by a Harvard infusion/withdrawal pump (Model 600-900). Peak drug vascular effects were calculated and expressed as percent changes in mean perfusion pressure from the pre-administration control levels; increase, vasoconstriction; decrease, vasodilatation.

\section{Miscellaneous}

SG-75, SG-86 (main metabolite of SG-75), and SG-89 (internal standard), chemical structures shown in Fig. 1, were all synthetized in Chugai Research Laboratories.

SG-75 and SG-86 vere in the form of a whitish crystalline powder, and the melting points were $87-93^{\circ} \mathrm{C}$ and $90-91^{\circ} \mathrm{C}$, respectively. SG-75 dissolves easily in acetic acid, ethyl alcohol, chloroform, ethyl acetate, and in water, whereas it does not dissolve readily in ethyl ether or in benzene. SG-86 is highly soluble in water, relatively soluble in ethyl acetate 
and not readily dissolved in ethyl ether or in benzene. 1\% aqueous solution of SG-75 is pH 6.3, while its pKa is 5.1. SG-75, SG-86 and SG-89 are stable in crystalline form, whereas their aqueous solutions are relatively unstable, at room temperature. SG-75, SG-86 and SG-89 added to rat or canine plasma were stable for at least 4 months, when stored at $-20^{\circ} \mathrm{C}$.

${ }^{14} \mathrm{C}$-Labeled SG-75 was synthesized by Dr. Tohira in Chugai Research Laboratories (Fig. 2). The specific activity is $5.75 \% \mathrm{Ci} / \mathrm{mg}$, and radiochemical purity $97.4 \%$.

Nitroglycerin, dissolved in $100 \%$ ethyl alcohol solution at a concentration of $40 \mathrm{mg} / \mathrm{ml}$, was provided by Nihon Kayaku Co., Tokyo.

\section{Statistical analysis}

Values in the text are represented as means \pm S.E., unless otherwise stated. The difference of paired mean values was analysed by the Student's $t$-test and considered significant when $P$ values were less than 0.05 .

\section{RESULTS}

\section{Metabolic Studies}

1. Plasma and urine levels of $S G-75$ and $S G-86$ following oral administration of $S G-75$ to the rat: As shown in Fig. 4., maximal plasma concentrations of SG-75 $(4.5 \mu \mathrm{g} / \mathrm{ml})$ and SG-86 $(3.6 \mu \mathrm{g} / \mathrm{ml})$ were attained at $30 \mathrm{~min}$ and $2 \mathrm{hr}$ after dosing, respectively, and decreased thereafter, the biological half life being $81 \mathrm{~min}$ and $227 \mathrm{~min}$, respectively.

In some experiments, ${ }^{14} \mathrm{C}-\mathrm{SG}-75(1 \mathrm{mg} / \mathrm{kg})$ was administered p.o. to 2 rats, and the $7 \mathrm{hr}$-urine samples were analyzed by a thin-layer chromatography. Results of one animal are shown in Fig. 5. SG-75 was metabolized in the body to at least 2 compounds; SG-86 in $62 \%$ of SG-75 administered and an unknown compound in $14 \%$, whereas SG-75, the parent compound, was scarcely detected. Similar results were obtained in the another animal.

2. Absorption and metabolism of SG-75 administered intraduodenally to vascularly perfused isolated rat small intestine: Experiments were performed on 15 preparations. At the beginning of perfusion, the flow rate was adjusted to give a mean perfusion pressure at about $100 \mathrm{mmHg}$. The perfusion pressure fell slightly and reached a steady-state level within $20 \mathrm{~min}$ of the onset of perfusion. At this stage, the measured parameters were as

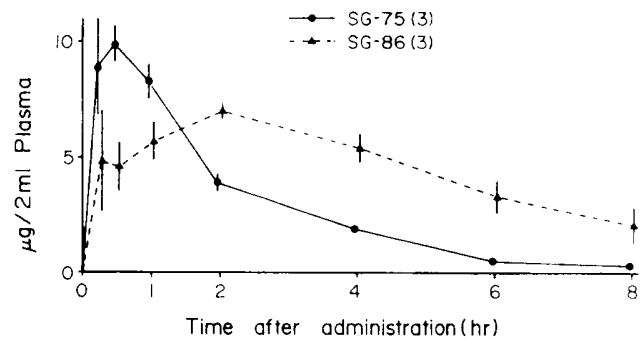

FIG. 4. Plasma concentration-time curves for SG-75 and its main metabolite SG-86 in the rat after oral administration of SG-75 $(5 \mathrm{mg} / \mathrm{kg})$. Vertical bars represent S.D. Number of experiments is given in parentheses. 


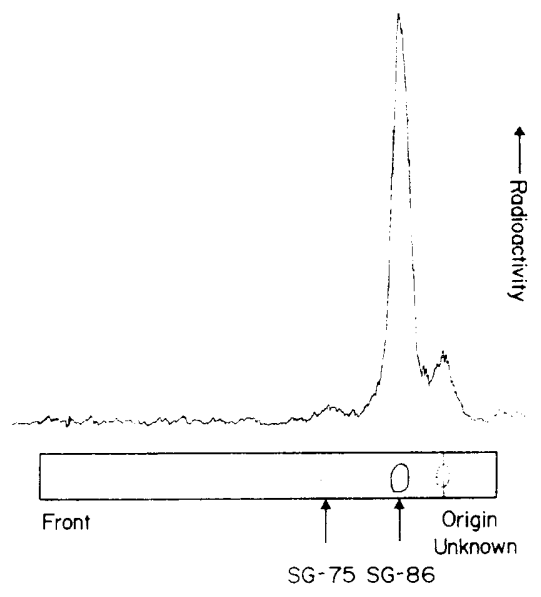

Fig. 5. Thin-layer chromatogram and radioautoscannogram of pooled urine collected from the rat after oral administration of ${ }^{1 .} \mathrm{C}-\mathrm{SG}-75 \quad(1 \mathrm{mg} / \mathrm{kg})$. Urine excreted over $7 \mathrm{hr}$ after ${ }^{14} \mathrm{C}-\mathrm{SG}-75$ administration was spotted on a thin-ayer plate in a volume of $0.02 \mathrm{ml}$ and developed in a solvent mixture of benzene/EtOH $(4: 1)$.

follows: mean mesenteric blood inflow, $3.8+0.1 \mathrm{ml} / \mathrm{min}(\mathrm{N}=15)$ and mean perfusion pressure, $97.8 \pm 3.5 \mathrm{mmHg}(\mathrm{N}=15)$.

Following dosing of SG-75 (100 $\mu \mathrm{g}, 500 \mu \mathrm{g}$ and $1 \mathrm{mg}$ as aqueous solution) inside the isolated intestine, the perfusion pressure gradually decreased during the first $5 \mathrm{~min}$, and a maximal steady-state level of the pressure was attained during the period of 10-20 min after administration: SG-75 $100 \mu$ g-treated group, $-16.9+2.3 \%, \mathrm{~N}=5 ; 500 \mu$ g-treated, $-23.1=$ $4.8 \%, \mathrm{~N}=5 ; 1 \mathrm{mg}$-treated, $-23.7 \pm 5.1 \%, \mathrm{~N}=5$. The arterial and venous blood samples were collected simultaneously and the absorption rate of SG-75 at appropriate intervals over one hour was obtained from the differences between the concentrations in arterial and venous plasma. As shown in Fig. 6, SG-75 was rapidly absorbed and transferred into the portal vein, while SG-86 was not detected in the circulating blood. One hour after administration of SG-75, the isolated intestine was removed from the perfusion apparatus and the

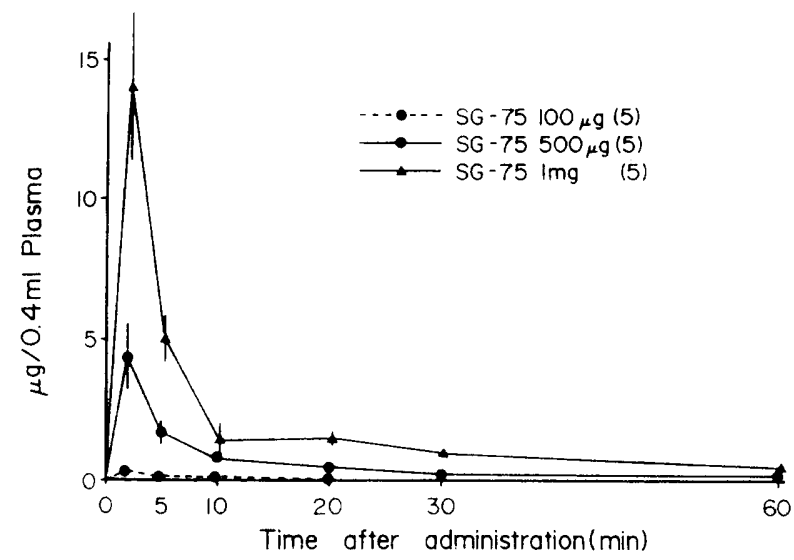

FIG. 6. The plasma concentration-time curves for SG-75 after intraduodenal administration of SG-75 in multiple doses to the vascularly perfused isolated rat small intestine. Vertical bars represent S.E. Number of experiments is given in parentheses. 
intestinal lumen was washed with $5 \mathrm{ml}$ of warmed $\left(37^{\circ} \mathrm{C}\right)$ distilled water. The recovery rates of SG-75 from the washed aqueous fraction of the isolated intestine were as follows: SG-75 $100 \mu$ g-treated group, $12.0 \pm 2.3 \mu \mathrm{g}, \mathrm{N}=5 ; 500 \mu \mathrm{g}$-treated, $81.4 \pm 21.4 \mu \mathrm{g}, \mathrm{N}=5$; 1 mg-treated, $104.9 \pm 26.8 \mu \mathrm{g}, \mathrm{N}=5$.

\section{Pharmacodynamic studies}

1. Influence of $S G-75$ and $S G-86$ on systemic blood pressure and heart rate in anesthetized intact rats

Single injections of SG-75 (0.03-1 mg/kg i.v.) decreased systemic blood pressure, in a dose-dependent manner. The hypotensive action of SG-75, in all doses tested, appeared at $30 \mathrm{sec}$ after injection, became maximal within $3 \mathrm{~min}$, and was accompanied by slight changes in the heart rate. Single doses $(0.3-10 \mathrm{mg} / \mathrm{kg})$ of SG-86 administered i.v. produced no significant changes in systemic blood pressure and heart rate. These data are summarized and depicted in Fig. 7.

2. Effects of SG-75 and SG-86 on isolated, donor-perfused rat hearts

2.1. Control observations: Experiments were carried out on 14 preparations. Mean perfusion pressure was set at a value slightly lower than the mean systemic blood pressure of the donor, at the onset of perfusion. Shortly after initiation of the perfusion, the pressure rose gradually to reach a steady-state level. The pressure was then re-adjusted to nearly $100 \mathrm{mmHg}$ and, thereafter, remained almost constant for about $3 \mathrm{hr}$. Thus, a stable situation of each preparation was established within 20-30 min of the onset of perfusion. The estimated main parameters from 14 preparations were as follows: left ventricular systolic pressure (LVP), $106 \pm 6 \mathrm{mmHg}$; heart rate, $247 \pm 10$ beats/min; mean perfusion pressure, $97 \pm 1 \mathrm{mmHg}$; coronary blood inflow, $3.1 \pm 0.1 \mathrm{ml} / \mathrm{min}$; mean systemic blood pressure of the donor, $110 \pm 3 \mathrm{mmHg}$.

2.2. Cardiovascular effects of SG-75 and SG-86: Single injections of SG-75 (0.3$30 \mu \mathrm{g})$ were given into the coronary perfusion system. SG-75 decreased perfusion pressure in a dose-dependent manner, but did not significantly influence LVP and heart rate. SG-86 had little effect on the cardiovascular system, although there was a slight decrease in perfusion

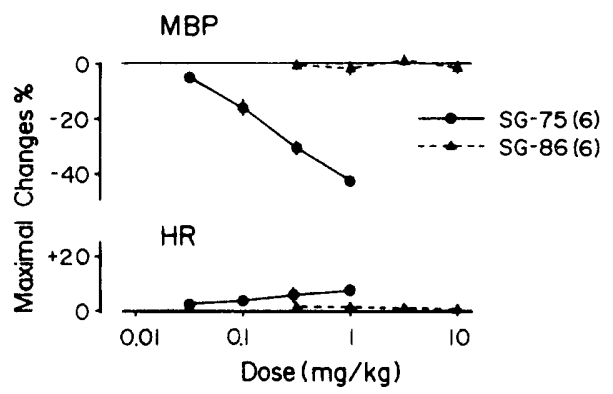

Fig. 7. Influence of SG-75 and SG-86 given i.v. on mean systemic blood pressure (MBP) and heart rate (HR) in rats. Vertical bars represent S.E., and number of experiments is given in parentheses. Initial values of MBP and HR were $121 \pm 3$ $\mathrm{mmHg}$ and $348 \pm 6$ beats/min $(\mathrm{N}=12)$, respectively. 


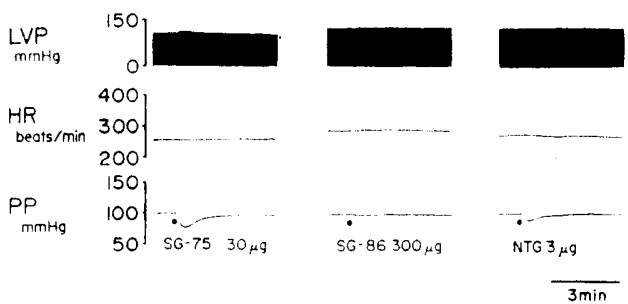

FIG. 8. Cardiac and vascular responses to SG-75 and SG-86 given i.a. into the isolated, donor-perfused rat heart. LVP, left ventricular systolic pressure; HR, heart rate: PP, mean perfusion pressure; NTG, nitroglycerin.

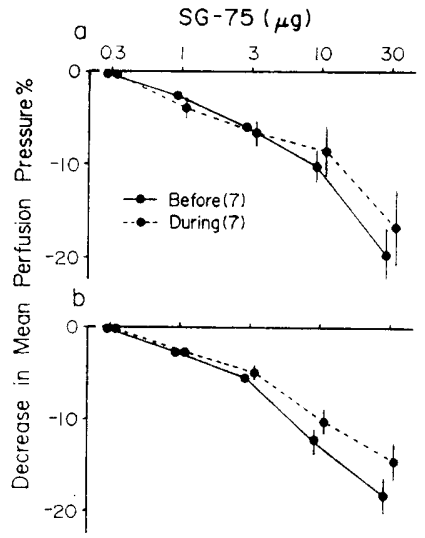

FIG. 9. Vascular effects of SG-75 given i.a. before and during the continuous infusion of SG-86 into the coronary perfusion system of the isolated, donor-perfused rat heart. Infusion rate of SG-86, a) $50 \mu \mathrm{g} / \mathrm{min}$ and b) $500 \mu \mathrm{g} / \mathrm{min}$. There were no significant differences between the corresponding values before ( - - $)$ and during (- ) the infusion of SG-86. Vertical bars represent S.E., and number of experiments is given in parentheses.

pressure $(-3.1 \pm 0.8 \%, \mathrm{~N}=4)$ with a large dose $(300 \mu \mathrm{g})$. Typical results are shown in Fig. 8 and the data are summarized in Fig. 9.

2.3. Influence of $S G-86$ on vascular responses to $S G-75$ : After control vascular responses to SG-75 were obtained, SG-86 was continuously infused into the coronary perfusion system at a rate of $50 \mu \mathrm{g} / 0.01 \mathrm{ml} \cdot \mathrm{min}^{-1}$ or $500 \mu \mathrm{g} / 0.02 \mathrm{ml} \cdot \mathrm{min}^{-1}$. Intra-arterial infusions of SG-86 produced a slight decrease in perfusion pressure (infusion rate $50 \mu \mathrm{g} / \mathrm{min},-5.8$ $\pm 2.0 \%, \mathrm{~N}=7 ; 500 \mu \mathrm{g} / \mathrm{min},-3.6 \pm 0.8 \%, \mathrm{~N}=7$ ) and no significant changes in LVP (infusuion rate $50 \mu \mathrm{g} / \mathrm{min}, 1.2 \pm 2.0 \%, \mathrm{~N}=6 ; 500 \mu \mathrm{g} / \mathrm{min}, 4.2 \pm 3.1 \%, \mathrm{~N}=7$ ). The gradual change of perfusion pressure following infusion of SG-86 reached a stable level within 5$10 \mathrm{~min}$ after the onset of infusion. Changes in vascular responses to SG-75 were then examined in the presence of SG-86, in the same preparation. As shown in Fig. 9, vascular responses to SG-75 were not significantly modified by continuous infusion of SG-86. 


\section{DISCUSSION}

Thin-layer chromatographic studies on the rat urine collected after oral dosing of ${ }^{14} \mathrm{C}-\mathrm{SG}-75$ indicated that SG-75 was converted mainly to SG-86, a denitrated product, in the rat. Also, SG-86 was recovered as a main metabolite in the urine of dogs and humans when a high pressure liquid chromatographic technique was used (our unpublished data). In both cases, SG-75 was scarcely detected. When SG-75 was administered into the intestinal lumen at the origin of duodenum in experiments using the vascularly perfused isolated rat small intestine, SG-75 was detected only in an unaltered form in the portal vein. This result clearly indicated that the administered SG-75 was rapidly absorbed through the intestinal wall over a wide range of concentrations, and the extent of denitration of SG-75 during absorption from the intestinal tract was negligible under the experimental conditions. Together with these findings and blood level estimation studies, conversion of SG-75 to its main metabolite, SG-86 was assumed not to occur in the intestinal lumen or tissue but rather to occur rapidly during the first passage of the drug through the liver.

In the present study in the rat, SG-75 revealed hypotensive and coronary vasodilating actions, but the main metabolite, SG-86, was pharmacologically inactive, even in large doses. Similar findings were also obtained in the dog (3). Thus, the nitrate moiety in the chemical structure of SG-75 seems to play an essential role in the development of pharmacological activity of this compound.

It should be noted that the peak of the plasma level of SG-75 appeared faster than that of SG-86, and that the plasma SG-86 was still at a high level while the plasma SG-75 decreased significantly. Thus, for the clinical application of SG-75, the question of whether or not SG-86 may modify the cardiovascular effects of SG-75 has to be considered. However, on the basis of the finding that coronary vasodilator responses to SG-75 administered intraarterially were not significantly altered by the continuous infusion of large doses of SG-86 into the coronary perfusion system, the above-mentioned possibility can be ruled out. All these results taken together indicate that when SG-75 is administered to rats, SG-86 formed in the liver, not in the intestinal lumen or tissue, as a main metabolite of the parent drug, becomes pharmacologically inactive and has no influence on the cardiovascular effect of SG-75. Further studies on the metabolic pathway of SG-75 to SG-86, including possibilities for the other pathways, are now under way.

\section{REFERENCES}

1) UChida, Y., Yoshimoto, N. ANd Murao, S.: Effect of 2-nicotinamidethyl nitrate (SG-75) on coronary circulation. Japan. Heart J. 19, 112-124 (1978)

2) Uchida, Y., Yoshimoto, N. ANd Murao, S.: Effects of anti-anginal agents on cyclical reductions of coronary blood flow. Japan. Heart J. 19, 904-912 (1978)

3) Taira, N., Satoh, K., Yanagisawa, T., Imai, Y. and Hiwatari, M.: Pharmacological profile of a new coronary vasodilator drug, 2-nicotinamidoethyl nitrate (SG-75). Clin. exp. Pharmacol. Physiol. 6, 301-316 (1979)

4) Nakagawa, Y., Takeda, K., Katano, Y., Tsukada, T., Kitagawa, T., Otori, T. and IMAI, S.: Effects of 2-nicotinamidoethyl nitrate on the cardiovascular system. Japan. Heart J. 6, 881-895 (1979)

5) Sakai, K., Akima, M., Hinohara, Y., Sasaki, M. And Niki, R.: Vascularly perfused rat 
small intestine: A research model for drug absorption. Japan. J. Pharmacol. 30, 231$241(1980)$

6) SAKAI, K.: Coronary vasoconstriction by locally administered acetylcholine, carbachol and bethanechol in isolated, donor-perfused, rat hearts. Brit. J. Pharmacol. 68, 625-632 (1980) 